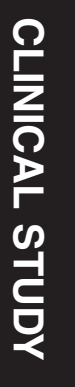

${ }^{1}$ Department of Ophthalmic Plastic and Reconstructive Surgery, Istanbul Beyoglu Eye Research and Training Hospital, Galata, Istanbul, Turkey

${ }^{2}$ Department of Ophthalmology, Baskent University, Ankara, Turkey

${ }^{3}$ Istanbul Beyoglu Eye Research and Training Hospital, Galata, Istanbul, Turkey

${ }^{4}$ Department of Ophthalmology, Baskent University, Istanbul, Turkey

${ }^{5}$ Faculty of Medicine, Department of Ophthalmology, Medeniyet University, Istanbul, Turkey

Correspondence: P Kaynak, Ophthalmic Plastic and Reconstructive Surgery Department, Istanbul Beyoglu Eye Research and Training Hospital, Kayali Bayir sok. 5/1, Kandilli, Istanbul 34688, Turkey

Tel: +90 532 3668738;

Fax: +90 2122916245 .

E-mail: pkaynak@gmail.com

Received: 20 April 2015 Accepted in revised form: 11 March 2016 Published online: 20 May 2016

This study had been presented in ASOPRS Fall symposium (14-15 November 2013) New Orleans, LA, USA.

\section{Comparison of botulinum toxin-A injection in lacrimal gland and conjunctivo- dacryocystorhinostomy for treatment of epiphora due to proximal lacrimal system obstruction}

P Kaynak ${ }^{1}$, GO Karabulut ${ }^{1}$, C Ozturker ${ }^{1}$, K Fazil ${ }^{1}$, YO Arat ${ }^{2}$, I Perente ${ }^{3}$, S Akar ${ }^{4}$, OF Yilmaz ${ }^{3}$ and A Demirok ${ }^{3,5}$

\author{
Abstract \\ Purpose To investigate and compare the \\ efficacy of botulinum toxin-A injection in the \\ lacrimal gland and conjunctivodacryocystor- \\ hinostomy surgery for the treatment of \\ epiphora caused by proximal lacrimal \\ system obstruction. \\ Methods Charts of the patients with \\ proximal canalicular obstruction who had \\ undergone conjunctivodacryocystorhinostomy \\ with permanent tube insertion (18 patients, \\ group 1) or 4 units of botulinum toxin-A \\ injection in the palpebral lobe of the lacrimal \\ gland (20 patients, group 2) were reviewed \\ retrospectively. The upper lacrimal system \\ obstruction was diagnosed by lacrimal system \\ irrigation. Schirmer-1 test and Munk epiphora \\ grading for evaluation of epiphora were \\ performed before the interventions and on \\ tenth day, first, third, and sixth months \\ after the interventions. \\ Results Improvement of epiphora was \\ statistically significant at all visits when \\ compared with values before injection \\ $(P<0.001)$ in both of groups. When two \\ techniques were compared, difference in \\ degree of epiphora before and after \\ intervention was not statistically significant \\ $(P<0.05)$. In group 2 , none of the patients had \\ punctate epitheliopathy, although there was a \\ significant decrease in Schirmer test results \\ $(P<0.001$, paired $t$-test). In group 1,9 cases \\ $(50 \%)$ had tube dislocation, 4 cases $(22.2 \%)$ \\ had obstruction, and granuloma \\ formation. Five cases $(25 \%)$ had ptosis \\ in group 2.
}

Conclusion Conjunctivodacryocystorhinostomy requires surgical experience, special postoperative care, and multiple revisions. As botulinum toxin-A injection in the lacrimal gland is technically easy, less-invasive, safe, with reversible effects, it can be considered as an alternative treatment in patients with proximal lacrimal system obstruction. Eye (2016) 30, 1056-1062; doi:10.1038/eye.2016.88; published online 20 May 2016

\section{Introduction}

Conjunctivodacryocystorhinostomy (C-DCR) with a Pyrex tube implantation was first described by Jones ${ }^{1}$ in 1965 . Since that time, C-DCR with permanent tube implantation has become the conventional approach for the management of canalicular obstruction. ${ }^{2}$ Upper lacrimal system dysfunction caused by obstruction can be due to congenital agenesis, herpetic infection, trauma, tumors, inflammation, lichen planus, Stevens-Johnson syndrome, systemic chemotherapy (especially docetaxel and 5-fluorouracil treatment), and radiation therapy but the cause of obstruction is mostly unknown. ${ }^{3-6}$ Because of the complications of C-DCR and tube implantation, such as extrusion (18-51\%), malposition (6-33\%), obstruction (11-47\%), infection, irritation, pain, and diplopia, patients can be dissatisfied in the long term ${ }^{3,6}$ and further interventions have to be performed to overcome these problems. 
The lacrimal gland is innervated by the cholinergic fibers of seventh cranial nerve. Injection of botulinum toxin-A (BTA) in the lacrimal gland decreases tear production by blocking presynaptic release of acetylcholine into neuromuscular end plates of cholinergic nerve fibers. ${ }^{7}$ Therefore injection of BTA in the lacrimal gland can be an alternative treatment in part for epiphora in lacrimal canalicular obstruction. Several reports about the use of BTA injection in the lacrimal gland for the treatment of gustatory hyperlacrimation, ${ }^{8-12}$ gustatory sweating (Frey's Syndrome), ${ }^{10}$ and functional epiphora ${ }^{13}$ have been published in the literature. BTA injections in patients with epiphora owing to obstruction of lacrimal apparatus have been reported in a few reports. ${ }^{4,8,14,15}$ In this article, we investigated and compared the efficacy of C-DCR and BTA injection in the lacrimal gland for treatment of epiphora owing to proximal lacrimal system obstruction.

\section{Subjects and methods}

A total of 38 eyes of 38 patients with epiphora as a result of canalicular obstruction who have $<8 \mathrm{~mm}$ of healthy proximal canaliculus were enrolled in this study and the charts of the patients were reviewed retrospectively. This study was a non-randomized, comparative study in which all patients were informed about the C-DCR procedure, off-label use of BTA, and the complications of both interventions and procedures were performed according to the patients' preferences. Informed consent was obtained from all patients in accordance with the Helsinki Declaration and the study was approved by the local ethics committee.

All patients underwent ophthalmic examination including visual acuity, external examination, slit lamp microscopy, and lacrimal syringing. The proximal lacrimal system obstruction, which consisted of the upper and lower canaliculi and common canaliculus, was diagnosed by lacrimal system irrigation to confirm complete obstruction and the decision for the treatment was made based on the shortage of residual healthy tissue for canalicular reconstruction.

Group 1 consisted of 18 eyes of 7 male and 11 female patients with a mean age of $45.5 \pm 14.3$ years who had C-DCR with permanent tube insertion between 2002 and 2012. Group 2 consisted of 20 eyes of 8 male and 12 female cases with a mean age of $49.7 \pm 12.9$ years that had injection of BTA in the lacrimal gland between 2009 and 2013. BTA injection in the palpebral lobe of lacrimal gland was performed instead of C-DCR in case of doubt about the patient's postoperative tube care, logistical problems (limited access for transportation, financial problems), refusal of primary lacrimal surgery or additional surgery for failed C-DCR by the patient and patients' poor surgical candidacy.

All C-DCR operations and injections of BTA were performed by single surgeon (PK).

In group 1, C-DCR with Metaireau tube insertion, which is a permanent silicone tube coated with poly-Nvinylpyrrolidinone (PVP), was performed with the traditional external approach that has been well described in the literature. ${ }^{2,3,6}$ Attention was paid to create the ostium as inferiorly as possible to enable easy drainage of tears down the tube.

In group 2, purified BTA (Botox; Allergan; Irvine, CA, USA: 100 mouse unit) injection in the lacrimal gland was performed under topical anesthesia with proparacain $0.5 \%$. BTA was diluted with $1 \mathrm{ml}$ of sterile, preservativefree saline (100 units $/ 1 \mathrm{ml}$ ). Upper eyelid was everted over Desmarres eyelid retractor. Patients were asked to look inferomedially and injection of 4 units of BTA, which was arbitrarily chosen under the guidance of previous studies, ${ }^{8-14}$ via 30 -gauge tuberculin syringe was performed in the palpebral lobe under direct visualization transconjunctivally. Tobramycin $0.2 \%$ eye drop was applied as a single dose after the injection. All patients were prescribed artificial teardrops three times daily and examined on the tenth day, first, third, and sixth months.

The patients were asked to subjectively rate their epiphora and evaluation was performed according to Munk Epiphora Grading System before and at tenth day, first, third, and sixth months follow-up visits ${ }^{16}$ (Table 1). To measure the baseline tear production, Schirmer- 1 test was performed (Tear-Flo, Odyssey Medical, Memphis, TN, USA) before BTA injection and at the tenth day, first, third, and sixth months follow-up visits after BTA injection by an independent masked observer. This testing was done at least $2 \mathrm{~h}$ after instillation of artificial tears.

Patients were divided into three groups according to their reduction in epiphora before and after injection of BTA in the lacrimal gland. Efficacy of BTA injection was analyzed in different age groups.

Table 1 Epiphora grading scale according to munk $^{7}$

\begin{tabular}{ll}
\hline 0 & No epiphora \\
1 & Occasional epiphora requiring drying or dabbing less than twice daily $(<2$ times) \\
2 & Epiphora requiring dabbing $2-4$ times daily $(2-4$ times) \\
3 & Epiphora requiring dabbing $5-10$ times daily (5-10 times) \\
4 & Epiphora requiring dabbing $>10$ times daily or constant tearing $(>10$ times) \\
\hline
\end{tabular}


For quantitative analysis Mann-Whitney $U$ test and for qualitative analysis $X^{2}$ test were used. When the requirements for $X^{2}$ test were not fulfilled, Fisher exact test was preferred instead. All statistical analyses were performed with IBM SPSS Statistics ver. 22 (IBM Corp., Armonk, NY, USA). The level of significance was accepted as $P<0.05$.

\section{Results}

There was no statistical difference in terms of age, sex, and side of the eye between group 1 and $2(P=0.098$, $P=0.94, P=0.78$ respectively; Table 2$)$. Length of followup in group 1 (13-132 months, median 100 months)

Table 2 Etiology of proximal lacrimal system obstruction

\begin{tabular}{llc}
\hline Etiology & $\begin{array}{c}\text { Group 1 no. } \\
(\%)\end{array}$ & $\begin{array}{c}\text { Group 2 no. } \\
(\%)\end{array}$ \\
\hline $\begin{array}{l}\text { Idiopathic } \\
\text { Trauma }\end{array}$ & $0(0)$ & $9(45)$ \\
Unsuccessful DCR with & $5(27.7)$ & $4(20)$ \\
recanalization & $9(50)$ & $3(15)$ \\
Congenital punctual agenesis & $0(0)$ & $2(10)$ \\
Lichen planus & $2(11.1)$ & $1(5)$ \\
Steven-Johnson syndrome & $0(0)$ & $1(5)$ \\
Systemic Lupus Erythematosus & $1(5.5)$ & $0(0)$ \\
Herpetic infection & $1(5.5)$ & $0(0)$ \\
\hline
\end{tabular}

was significantly longer than group 2 (6-53 months, median 6 months $)(P<0.001)$.

In group 1 the most common etiology was unsuccessful recanalization of the canaliculi and DCR (9 eyes, 50\%), which was followed by trauma $(27.7 \%)$. Etiology of canalicular obstruction was unknown in 9 eyes (45\%) in group 2. Trauma (20\%) and unsuccessful C-DCR (15\%) were other common causes (Table 2).

In patients who had C-DCR with tube implantation (group 1), the mean Munk score of patients was 4 before surgery. Improvement of epiphora according to the mean Munk scores was statistically significant on the tenth day, first, third, and sixth months $(1.83,1,1.22$, and 1.5 , respectively) $(P<0.0001)$ when compared with values before the operation. In patients who were injected BTA in the lacrimal gland (group 2) the mean Munk score was 3.95 before BTA injection. The improvement of epiphora was also statistically significant on each visit where the mean Munk scores were 1.6 on the tenth day, 1.05 at first month, 1.36 at third month, and 1.6 at sixth month $(P<0.0001)$. There was no significant difference in epiphora grading before the interventions between two groups. After the interventions, the improvement in epiphora was not statistically significant between group 1 and 2 (Figure 1). BTA injection in the lacrimal gland was still significantly effective in the improvement of epiphora at sixth month follow-up visit.

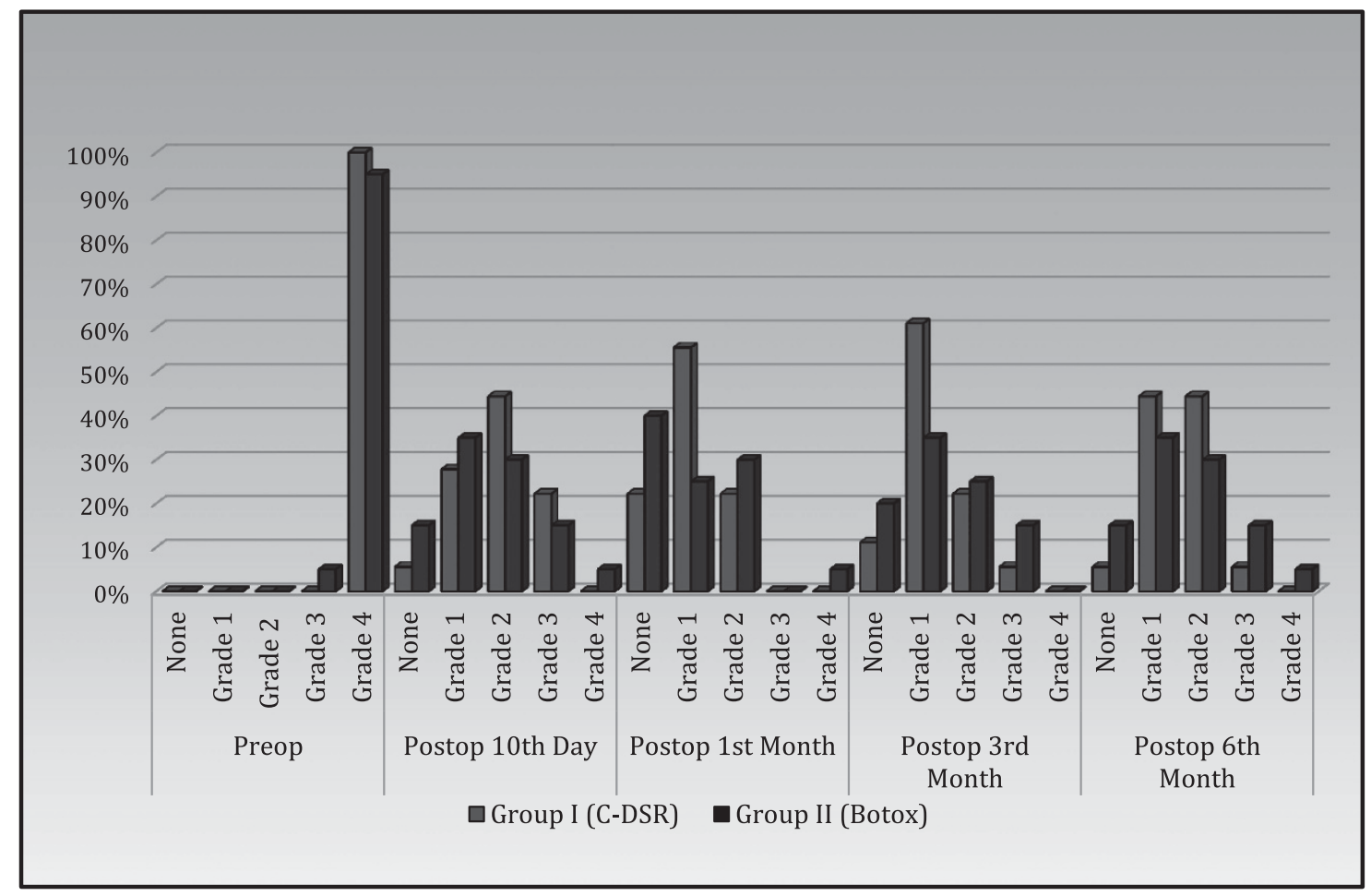

Figure 1 Epiphora grading of cases before and after interventions. 
Before BTA injection in the lacrimal gland mean Schirmer-1 test result was $12 \pm 0.27 \mathrm{~mm}$ in group 2 , which declined to $8.6 \pm 0.2 \mathrm{~mm}, 5.4 \pm 0.16 \mathrm{~mm}, 5.8 \pm 0.27 \mathrm{~mm}$, and $9.8 \pm 0.43 \mathrm{~mm}$ on the 10th day, first, third, and sixth months follow-up visits, respectively. Compared to the pre-injection values, the reduction in the Schirmer-1 test results was statistically significant at each visit after the procedure $(P<0.001)$. Despite the decrease in Schirmer test results, the patients did not develop symptoms of dry eye or corneal staining on examination.

To investigate the efficacy of BTA injection in the lacrimal gland in different age groups, the patients were grouped according to their decrease in the Munk epiphora grading before and after the intervention. There was no correlation between change in degree of epiphora and ages of the patients $(P=0.48)$.

Thirteen of our patients had 6-8 months follow-up. The patients did not need reinjection during this period. Seven patients had 24-53 months follow-up. Two patients requested repeated injections. BTA treatment was repeated four times in each eye of one case and twice in one eye of one case as a result of patients' high satisfaction with the treatment. The longer follow-up after the multiple injections of these two patients were 53 and 36 months, respectively. The remaining five patients were happy with the results; however, they could not receive multiple injections owing to social inconveniences such as travel and injection costs. The duration of epiphora-free period varied between 6 and 12 months in these patients.

In group 1, tube dislocation was seen in 9 cases (50\%), obstruction and granuloma formation was seen in 4 cases (22.2\%). In one patient although C-DCR surgery was uneventful, it was unsuccessful in treating symptoms despite eight revisions due to tube dislocation and therefore the patient chose to be treated with BTA, which successfully treated the epiphora and had repeated injections.

In group 2, 5 cases (25\%) had blepharoptosis that resolved in 2-3 weeks after injection of BTA. Two of them who had ptosis $>1 \mathrm{~mm}$ were prescribed apraclonidine hydrochloride $0.5 \%$ drops PRN to manage ptosis during this period.

\section{Discussion}

Epiphora caused by proximal lacrimal system obstruction was the main indication for C-DCR operation and BTA injection in the lacrimal gland in this study. It has been suggested that the presence of $<8 \mathrm{~mm}$ of healthy proximal canaliculus or the failure of orbicularis oculi muscle and the tear pump needs intervention. 3,6,17 Lim $e a^{3}$ reported that the cause of obstruction was mostly idiopathic and trauma was the most common cause where the etiology was known. Consistent with previous studies, $3,6,18-20$ idiopathic obstruction, trauma, and unsuccessful lacrimal surgery were the most common causes of obstruction in our study as well. Lichen planus, congenital agenesis, systemic lupus erythematosus, Stevens-Johnson syndrome, and herpetic infection were the other less common causes.

In conventional approach, many surgeons prefer to perform C-DCR with tube implantation for lacrimal canalicular obstruction. ${ }^{2,3,6,17-23}$ Extrusion rate was $50 \%$ in this study, which had been reported as $18-51 \%$ 2,3,6,18,20 in the literature. To overcome this problem PuttermanGladstone tubes with additional flange, ${ }^{3,6,21}$ frosted Jones pyrex tubes with textured outer surface,,$^{2,3,22}$ and porouspolyethylene-coated tubes ${ }^{3,23}$ were introduced to increase the stability in the surgical fistula. Rose and Welham reported a step-wise increase in the Jones tube replacement or repositioning over time and estimated that $100 \%$ of cases would require these procedures if observed over 20 years. ${ }^{17}$ So they suggested surgical tube replacement should perhaps be regarded as a part of expected maintenance, rather than as a complication. In this study, Metaireau tubes were preferred and were sutured around their flange to caruncle area to overcome extrusion and dislocation. Metaireau tube is a silicone tube coated with PVP. The PVP coating makes the surface as slick as glass to enhance tear drainage, prevents debris from collecting on the surface and provides the properties of flexibility, elasticity, and anti-adherence. ${ }^{24}$ However, extrusion and malposition were the main reasons of our patients' dissatisfaction with the surgery.

Obstruction of the tube was another complication in our study $(22.2 \%)$. To overcome this problem all the patients were advised regular sniffing, cleaning of the entrance of the tube with cotton swab to remove mucus and aspirating water or saline forcefully to maintain tube patency as described in previous reports. 3,6,17 Most common cause of obstruction was soft tissue hypertrophy or granuloma formation in this study despite cautery of caruncle or redundant conjunctiva in the primary operation. In the study by Lim et $a l^{3}$ a total of 37 cases were asked about their level of satisfaction with the C-DCR and 11 of them were dissatisfied due to the cost of a repeat procedure and trouble of maintaining the tube with daily cleaning and eye baths to remove mucus plugs. According to Rosen et $a l^{19}$ the reasons for patient dissatisfaction in $11.6 \%$ of successfully treated patients were the number of follow-up examinations, problems in tube maintenance, tearing in recumbent position, fogging and spraying spectacles, and esthetics. Although there was a statistically significant decrease in epiphora after C-DCR operation half of our patients were dissatisfied due to complications and high number of follow-up examinations. 
BTA injection to control hypersecretion from cholinergically supplied lacrimal gland was reported as an alternative treatment for epiphora. ${ }^{4,7,8}$ In 1998 Boroojerdi et al ${ }^{9}$ published the first report about the use of BTA for the treatment of epiphora in patients with gustatory hyperlacrimation (Crocodile Tears Syndrome) secondary to peripheral facial nerve palsy. He reported that BTA injection into orbicularis oculi for involuntary synkinesia coincidentally treated symptoms of reflex hyperlacrimation. Several other reports about the use of BTA injection in the lacrimal gland for the treatment of gustatory hyperlacrimation, ${ }^{8-12}$ gustatory sweating (Frey's Syndrome), ${ }^{10}$ and functional epiphora ${ }^{13}$ have been published in the literature. However, there are a few reports that indicate injection of BTA in the lacrimal gland for lacrimal apparatus obstruction. ${ }^{4,8,14,15}$ BTA injection in the lacrimal gland is devoid of the risks and complications of an invasive surgery and general anesthesia. Injection can be performed in an outpatient clinic. This technique is easy, minimally invasive, and less time consuming, compared with surgery. However, its effect is transient and side effects such as dry eye, ptosis, and diplopia can be seen with this off-label use of BTA.4,8-14

Transconjunctival injection in the palpebral lobe of the lacrimal gland is usually preferred due to the ability to directly visualize the lacrimal gland during injection. ${ }^{4,7,8,11-14,25-27}$ However, transcutaneous injection in the orbital lobe was also reported. ${ }^{9,10,28}$ Montoya et al ${ }^{12}$ injected 20 U Dysport initially via transcutaneous route but later progressed to transconjunctival route because of the confidence that the entire amount of BTA was directly delivered to the lacrimal gland. A meta-analysis reported by Falzon $e a^{25}$ revealed that transconjunctival approach was associated with fewer complications. In the study of Nava-Castaneda, it is suggested that the intraglandular injection via transconjunctival approach allowed the use of a minimum dose of BTA. ${ }^{26}$

Several different doses and concentrations of BTA injection have been published in the literature (2.5-60 U). ${ }^{4,7-14,25-28}$ Higher doses seem to have no additional benefit in terms of efficacy and duration. 7,9 Wojno reported $63 \%$ improvement with $2.5 \mathrm{U}$ of BTA in patients with lacrimal obstruction, but $37 \%$ needed an additional $2.5 \mathrm{U}$. For these patients who required a total of $5 \mathrm{U}$ of BTA, the entire dose was given in one injection on subsequent visits. ${ }^{8}$ Ziahossein et al ${ }^{14}$ recommended a starting dose of 2.5 units of BTA in their recent study. In our study, $4 \mathrm{U}$ of BTA was injected transconjunctivally and was found to be effective in the treatment of epiphora.

Broojerdi suggested that the effect of BTA injection on hyperlacrimation seemed to be more stable than the effect on synkinesias. ${ }^{9}$ Usually the effect of BTA subsides within 2-4 months because of the collateral sprouting of nerve endings in muscles, but this may take much longer within the autonomic nervous system. $4,9,10,12$ The effect was found to be $4-5$ months in the study of Hofmann et al ${ }^{10}$ Nava-Castaneda et $a l^{26}$ reported the BTA effect up to 24 weeks when injected in the lacrimal gland. In this study, BTA injection in the lacrimal gland was still significantly effective in the improvement of epiphora at sixth month follow-up visit, which was similar to the previous studies. ${ }^{4,9,10,12,26}$

The most common complications of BTA injection in the lacrimal gland are blepharoptosis, diplopia, lagophthalmus, conjunctivitis, and dry eye. ${ }^{4,7,9-13,25-28}$ Demetriades et al ${ }^{7}$ investigated the relationship among administered dose, reduced tear production, and associated side effects in an animal model. They injected $0.1 \mathrm{ml} 0.625 \mathrm{U}, 1.25 \mathrm{U}$ or 2.5U BTA transconjunctivally in the lacrimal gland of one eye of white female rabbits and $0.1 \mathrm{ml}$ of $0.9 \%$ sodium chloride in the contralateral lacrimal gland. They found mild blepharoptosis in some rabbits, but the incidence was not different between the BTA injected and the control eyes. Whittaker et al ${ }^{13}$ reported ptosis and transient diplopia in 2 out of 14 patients with functional epiphora, both of whom received $5 \mathrm{U}$. They did not see any side effect with 2.5U BTA injections. Nava-Castaneda et $a l^{26}$ reported 2 out of 18 patients had transient ptosis with $2.5 \mathrm{U}$ transconjunctival injections. Temporary ptosis was also seen in $11 \%$ of the patients in the study of Wojno ${ }^{8}$ that resolved within 1-3 weeks. Only one patient had ptosis after injection of 2.5U BTA, whereas 4 patients had ptosis after a total of $5 \mathrm{U}$ BTA, suggesting that ptosis was dose related due to diffusion to the levator muscle. Ziahossein et al ${ }^{14}$ reported lacrimal gland and upper eyelid hematoma, and horizontal diplopia both of which lasted for 2 weeks. In our study, five patients (25\%) had ptosis, which resolved in 2-3 weeks. In one patient ptosis occurred following 2 out of 4 injections. Since we use the same dose $(4 \mathrm{U})$ and the same relatively small volume $(0.04 \mathrm{ml})$ for all injections, diffusion of BTA into levator muscle in two out of four applications for this patient might be due to the injection technique.

Main and accessory lacrimal glands contribute to the production of the aqueous layer of the tear film via reflex and basal tear production mechanisms. Injections of BTA in the lacrimal gland have been shown to decrease the excess tearing in many studies. ${ }^{4,7-15,25-28}$ Demetriades et $\mathrm{al}^{7}$ reported significant decrease in tear production with 1.25 and 2.5U BTA injections compared with the contralateral eye injected with saline but observed no evidence of dry eye or corneal pathology after the application of either fluorescein or Rose Bengal staining. In the study of Boroojerdi et al ${ }^{9}$ after injection of BTA transcutaneously in the lacrimal gland no patient showed dry eye confirmed by Schirmer test. Montoya et al ${ }^{12}$ 
reported 2 patients with dry eye symptoms for 2 months both of which were easily controlled with artificial tears. In the study of Nava-Castaneda et $a l^{26}$ there were statistically significant differences in all Schirmer test values after BTA injection compared with those before injection. They observed positive corneal staining with Lissamine green that did not worsen during the study suggesting that the applied 2.5U BTA dose did not cause any complication by decreasing the tear volume.

Similarly, in our study the decrease in Schirmer test results were statistically significant when compared with pre-injection values. However, none of the patients had corneal staining or punctuate epitheliopathy with fluorescein staining during this study. It can be presumed that the dry eye complications might have been overcomed with the help of artificial tears in our study.

With aging both tear production and symptoms of epiphora may decrease. ${ }^{29,30}$ Ziahossein et al ${ }^{14}$ reported that majority of their patients who benefited from BTA injection were elderly and BTA was a useful alternative in this age group. We wanted to investigate whether the effect of BTA injection in the lacrimal gland increases in older age groups due to decreased tear production. However, there was no correlation between age of patients and decrease in epiphora after intervention.

In children epiphora secondary to proximal lacrimal system obstruction is difficult to treat with C-DCR and permanent tube implantation because of the need for a special postoperative care, the low patient compliance and the higher rate of postoperative complications. Eustis and Babiuch injected BTA in the lacrimal gland of 3 children over a 2-year period and the patients were treated with repeated BTA injections when epiphora returned. ${ }^{15}$ They observed partial or full relief of symptoms within 2-8 weeks lasting 6-13 months without any complication. So, BTA injection in the lacrimal gland can be an alternative treatment in children with canalicular obstruction. We did not perform injections of BTA in children in this study.

In conclusion, although C-DCR is widely used in the treatment of proximal lacrimal system obstructions with success, it requires surgical experience, special postoperative care, necessitates multiple revisions and is contraindicated in patients who are medically frail or unable to comprehend the treatment, are unable to attend for follow-up and those with previous malignancy involving the lacrimal system. The results of our study are in accordance with previous studies, while it proves that BTA injection in the lacrimal gland avoids the risks and complications of an invasive surgery and general anesthesia and the injection can be performed in an outpatient clinic. BTA injection in lacrimal gland can be considered as a viable option in treatment of proximal lacrimal system obstructions, in case of poor surgical candidacy and unwillingness to have surgery.

Summary

What was known before

- In conventional approach, many surgeons prefer to perform conjunctivodacryocystorhinostomy with tube implantation for lacrimal canalicular obstruction.

What this study adds

- Botulinum toxin-A injection in lacrimal gland, which is a relatively easy technique avoiding the risks and complications of an invasive surgery and general anesthesia, can be considered as an alternative method in treatment of proximal lacrimal system obstructions.

\section{Conflict of interest}

The authors declare no conflict of interest.

\section{References}

1 Jones LT. Conjunctivodacryocystorhinostomy. Am J Ophthalmol 1965; 59: 773-783.

2 Steele EA, Dailey RA. Conjunctivodacryocystorhinostomy with the frosted Jones Pyrex tube. Ophthal Plast Reconstr Surg 2009; 25: 42-43.

3 Lim C, Martin P, Benger R, Kourt G, Ghabrial R. Lacrimal canalicular bypass surgery with the Lester Jones tube. Am J Ophthalmol 2004; 137: 101-108.

$4 \mathrm{Tu} \mathrm{AH}$, Chang EL. Botulinum toxin for palliative treatment of epiphora in a patient with canalicular obstruction. Ophthalmology 2005; 112: 1469-1471.

5 Haidak DJ, Hurwitz BS, Yeung KY. Tear-duct fibrosis (dacryostenosis) due to 5-fluorouracil. Ann Intern Med 1978; 88: 657.

6 Athanasiov PA, Madge S, Kakizaki H, Selav D. A review of bypass tubes for proximal lacrimal drainage obstruction. Surv Ophthalmol 2011; 56: 252-266.

7 Demetriades AM, Leyngold IM, D'Anna S, Erghari AO, Emmert DG, Grant MP et al. Intraglandular injection of Botulinum Toxin A reduces tear production in rabbits. Ophthal Plast Reconstr Surg 2013; 29: 21-24.

8 Wojno TH. Results of lacrimal gland botulinum toxin injection for epiphora in lacrimal obstruction and gustatory tearing. Ophthal Plast Reconstr Surg 2011; 27: 119-121.

9 Boroojerdi B, Ferbert A, Schwarz M, Herath H, Noth J. Botulinum toxin treatment of synkinesia and hyperlacrimation after facial palsy. J Neurol Neurosurg Psychiatry 1998; 65: 111-114.

10 Hofmann JR. Treatment of Frey's Syndrome (gustatory sweating) and 'Crocodile Tears' (gustatory epiphora) with purified botulinum toxin. Ophthal Plast Reconstr Surg 2000; 16(4): 289-291.

11 Baranano DE, Miller NR. Long term efficacy and safety of botulinum toxin A injection for crocodile tears syndrome. Br J Ophthalmol 2004; 88(4): 588-589.

12 Montoya FJ, Riddell CE, Caesar R, Hague S. Treatment of gustatory hyperlacrimation (crocodile tears) with injection of 
botulinum toxin into the lacrimal gland. Eye 2002; 16: 705-709.

13 Whittaker KW, Matthews BN, Fitt AW, Sandramouli S. The use of botulinum toxin A in the treatment of functional epiphora. Orbit 2003; 22(3): 193-198.

14 Ziahosseini K, Al-Abbadi Z, Malhotra R. Botulinum toxin injection for the treatment of epiphora in lacrimal outflow obstruction. Eye (Lond) 2015; 29(5): 656-661.

15 Eustis HS, Baiuch A. Use of botulinum toxin injections to the lacrimal gland for epiphora in children with proximal obstruction of lacrimal drainage system. J Pediatr Ophthalmol Strabismus 2012; 16(1): e15-e16.

16 Munk PL, Lin DT, Morris DC. Epiphora: treatment by means of dacryocystoplasty with baloon dilatation of the nasolacrimal drainage apparatus. Radiology 1990; 177(3): 687-690.

17 Rose GE, Welham RA. Jones' lacrimal canalicular bypass tubes: twenty-five years' experience. Eye (Lond) 1991; 5(Pt 1): 13-19.

18 Zilelioğlu G, Gunduz K. Conjunctivodacryocystorhinostomy with Jones tube. A 10-year study. Doc Ophthalmol 1996; 92: 97-105.

19 Rosen N, Ashkenazi I, Rosner M. Patient dissatisfaction after functionally successful conjunctivodacryocystorhinostomy with Jones Tube. Am J Ophthalmol 1994; 117: 636-642.

20 Sekhar GC, Dortzbach RK, Gonnering RS, Lemke BN. Problems associated with conjunctivodacryocystorhinostomy. Am J Ophthalmol 1991; 112: 502-506.
21 Gladstone GJ, Putterman AM. A modified glass tube for conjunctivodacryocystorhinostomy. Arch Ophthalmol 1985; 103: 1229-1230.

22 Dailey RA, Tower RN. Frosted Jones pyrex tubes. Ophthal Plast Reconstr Surg 2005; 21: 185-187.

23 Wojno T. Experience with a Medpor-coated tear drain. Ophthal Plast Reconstr Surg 2010; 26: 327-329.

24 Chang CH, Chen YC, Lee CL, Chu SW. Application of the Metaireau tube (M-tube) in conjunctivodacryocystorhinostomy (CDCR). Taiwan J Ophthalmol 2013; 3: 71-74.

25 Falzon K, Galea M, Cunniffe G, Logan P. Transconjunctival botulinum toxin offers an effective, safe and repeatable method to treat gustatory lacrimation. Br J Ophthalmol 2010; 94: $379-380$.

26 Nava-Castaneda A, Tovilla-Canales JL, Boullosa V, Tovilla-y-Pomar JL, Monroy-Serrano MH, Tapia-Guerra V et al. Duration of botulinum toxin effect in the treatment of crocodile tears. Ophthal Plast Reconstr Surg 2006; 22(6): 453-456.

27 Riemann R, Pfennigsdorf S, Riemann E, Naumann M. Successful treatment of crocodile tears by injection of botulinum toxin into the lacrimal gland: a case report. Ophthalmology 1999; 106: 2322-2324.

28 Yavuzer R, Basterzi Y, Akata F. Botulinum toxin A for the treatment of crocodile tears. Plast Reconstr Surg 2011; 27: 119-121.

29 Guillon M, Maïssa C. Tear film evaporation-effect of age and gender. Cont Lens Anterior Eye 2010; 33(4): 171-175.

30 Nanavaty MA, Vasavada AR, Gupta PD. Dry eye syndrome. Asian J Exp Sci 2006; Vol. 20, Supplement 63-80. 